\title{
A note on optimal Hermite interpolation in Sobolev spaces
}

\author{
Guiqiao $X u^{1 *}$ (D) and Xiaochen $Y u^{1}$
}

\section{"Correspondence:}

Xuguiqiao@aliyun.com

'Department of Mathematics, Tianjin Normal University, Tianjin, 300387, P.R. China

\begin{abstract}
This paper investigates the optimal Hermite interpolation of Sobolev spaces $W_{\infty}^{n}[a, b]$, $n \in \mathbb{N}$ in space $L_{\infty}[a, b]$ and weighted spaces $L_{p, \omega}[a, b], 1 \leq p<\infty$ with $\omega$ a continuous-integrable weight function in $(a, b)$ when the amount of Hermite data is $n$. We proved that the Lagrange interpolation algorithms based on the zeros of polynomial of degree $n$ with the leading coefficient 1 of the least deviation from zero in $L_{\infty}$ (or $\left.L_{p, \omega}[a, b], 1 \leq p<\infty\right)$ are optimal for $W_{\infty}^{n}[a, b]$ in $L_{\infty}[a, b]$ (or $L_{p, \omega}[a, b]$, $1 \leq p<\infty)$. We also give the optimal Hermite interpolation algorithms when we assume the endpoints are included in the interpolation systems.
\end{abstract}

MSC: $41 \mathrm{~A} 05 ; 41 \mathrm{~A} 25 ; 41 \mathrm{~A} 46$

Keywords: Optimal Hermite interpolation; Sobolev space; Worst-case setting

\section{Introduction and main results}

Let $F$ be a Banach space of functions defined on a compact set $D$ that can be continuously embedded in $C^{r}(D), B F$ be the unit ball of $F$, and $G(\supseteqq F)$ be a normed linear space with norm $\|\cdot\|_{G}$. We want to approximate functions $f$ from $F$ by using a finite number of arbitrary Hermite data $f^{(s)}(t)$ for some $s \leq r$ and $t \in D$. We use an algorithm $A_{n}$ that uses exactly $n$ Hermite data to reconstruct functions from $B F$. The worst-case error of the algorithm $A_{n}$ for $B F$ in $G$ is defined by

$$
e\left(B F, A_{n}, G\right):=\sup _{f \in B F}\left\|f-A_{n}(f)\right\|_{G} .
$$

Let $\Lambda_{n}$ be an algorithm class that uses exactly $n$ Hermite data, $\Lambda=\bigcup_{n=1}^{\infty} \Lambda_{n}$. If there exists an algorithm $A_{n}^{*} \in \Lambda_{n}$ such that

$$
e\left(B F, A_{n}^{*}, G\right)=\inf _{\Lambda_{n}} e\left(B F, A_{n}, G\right)
$$

then we call $A_{n}^{*}$ the $n$th optimal algorithm for $\Lambda$ in the norm $G$. The value $e\left(B F, A_{n}^{*}, G\right)$ is called the $n$th optimal worst-case error for $B F$ in $G$ and we denote it as $e(n, B F, G)$.

(c) The Author(s) 2022. This article is licensed under a Creative Commons Attribution 4.0 International License, which permits use, sharing, adaptation, distribution and reproduction in any medium or format, as long as you give appropriate credit to the original author(s) and the source, provide a link to the Creative Commons licence, and indicate if changes were made. The images or other third party material in this article are included in the article's Creative Commons licence, unless indicated otherwise in a credit line to the material. If material is not included in the article's Creative Commons licence and your intended use is not permitted by statutory regulation or exceeds the permitted use, you will need to obtain permission directly from the copyright holder. To view a copy of this licence, visit http://creativecommons.org/licenses/by/4.0/. 
Let $L_{\infty} \equiv L_{\infty}[a, b]$ be the space of measurable functions defined on $[a, b]$, for which the norm

$$
\|f\|_{\infty}:=\operatorname{ess~sup}_{x \in[a, b]}|f(x)|
$$

is finite. Meanwhile, for $1 \leq p<\infty$ and continuous-integrable $\omega(x)>0$ on $(a, b)$, let $L_{p, \omega} \equiv$ $L_{p, \omega}[a, b]$ be the space of measurable functions defined on $[a, b]$, for which the norm

$$
\|f\|_{p, \omega}:=\left(\int_{a}^{b}|f(x)|^{p} \omega(x) d x\right)^{1 / p}
$$

is finite. Using $C^{n} \equiv C^{n}[a, b], n=0,1,2, \ldots$ represents the spaces of functions with an $n$ thorder continuous derivative on $[a, b]$, respectively. Denote by $W_{\infty}^{n} \equiv W_{\infty}^{n}[a, b], n \in \mathbb{N}$ the class of all functions $f$ such that $f^{(n-1)}\left(f^{(0)}:=f\right)$ are absolutely continuous and $f^{(n)} \in L_{\infty}$.

For the construction of algorithms for approximating multivariate functions using function values, the univariate Lagrange interpolation polynomial algorithms play a key role, see $[2,5,8,13-15]$. Recently, some papers $[3,10]$ have considered the algorithms for approximating multivariate functions using Hermite data. To compare the approximation errors of Lagrange interpolation and Hermite interpolation, we introduce the concept of Hermite interpolation.

Let $x_{1}, x_{2}, \ldots, x_{r}$ be $r$ distinct points in $[a, b]$. Let $\Delta:=\left\{a \leq x_{1}<x_{2}<\cdots<x_{r} \leq b, \alpha_{i} \in\right.$ $\left.\mathbb{N}, n=\sum_{i=1}^{r} \alpha_{i}\right\}$ be a Hermite interpolation system. Then, the Hermite interpolation polynomial $H_{\Delta}(f)$ of a function $f \in W_{\infty}^{n}$ based on $\Delta$ is defined by

$$
H_{\Delta}(f) \in \mathcal{P}_{n-1}, \quad \text { and } \quad H_{\Delta}^{(j)}\left(f, x_{i}\right)=f^{(j)}\left(x_{i}\right), \quad 0 \leq j \leq \alpha_{i}-1,1 \leq i \leq r,
$$

where, and in the following, $\mathcal{P}_{n}$ represents the space of all algebraic polynomials of degree at most $n$. The classical Hermite interpolation formula gives

$$
H_{\Delta}(f, x)=\sum_{k=1}^{r} \frac{W_{\Delta}(x)}{\left(x-x_{k}\right)^{\alpha_{k}}} \sum_{h=0}^{\alpha_{k}-1} f^{(h)}\left(x_{k}\right) \frac{\left(x-x_{k}\right)^{h}}{h !}\left\{\frac{\left(x-x_{k}\right)^{\alpha_{k}}}{W_{\Delta}(x)}\right\}_{\left(x_{k}\right)}^{\left(\alpha_{k}-h-1\right)} \text {, }
$$

where, and in the following,

$$
W_{\Delta}(x)=\prod_{k=1}^{r}\left(x-x_{k}\right)^{\alpha_{k}}
$$

and $\{f(x)\}_{\left(x_{k}\right)}^{(s)}$ is the $s$-degree Taylor polynomial of $f$ at $x_{k}$. In particular, if $x_{1}, x_{2}, \ldots, x_{n}$ are $n$ distinct points in $[a, b]$, i.e., $\Delta:=\left\{a \leq x_{1}<x_{2}<\cdots<x_{n} \leq b, \alpha_{i}=1, i=1,2, \ldots, n\right\}$, then we obtain the well-known Lagrange interpolation

$$
L_{\Delta}(f, x)=\sum_{k=1}^{n} f\left(x_{k}\right) \ell_{k}(x)
$$

where

$$
\ell_{k}(x)=\frac{W_{\Delta}(x)}{\left(x-x_{k}\right) W_{\Delta}^{\prime}\left(x_{k}\right)}, \quad k=1,2, \ldots, n .
$$


Choosing interpolation systems is important for interpolation algorithms. For example, given a sufficiently smooth function, if nodes are not suitably chosen, then the Lagrange interpolation polynomials do not converge to the function as the number of nodes tends to infinity. A well-known example is Runge's phenomenon. Hence, the study of optimal Lagrange interpolation nodes is a hot research topic, see $[1,6,9,16]$ and the references therein.

The most important optimal Lagrange interpolation nodes problem is for $C^{0}$ in $L_{\infty}$. For $n=3$ and $n=4$, the results can be found in [11] and [12], respectively. For $n \geq 5$, it is still an open problem. For $r \geq 1$, it is well known that the $r$ th optimal Lagrange interpolation nodes are the zeros of the $r$ th Chebyshev polynomial of the first kind for $C^{r}[-1,1]$ in $L_{\infty}[-1,1]$. Recently, [16] obtained the $r$ th optimal Lagrange interpolation nodes for $W_{\infty}^{r}[-1,1]$ in $L_{p, \omega}[-1,1], 1 \leq p<\infty$.

Hermite interpolation is a kind of interpolation that is wider than Lagrange interpolation. It can use not only the function value data but also the derivatives value data. Under the condition of using the same amount of data, can increasing the use of derivatives value data make the calculation result more accurate? In general the answer is no. In the following, we give the optimal Hermite interpolation systems to show this.

Let

$$
E_{n, p, \omega}:=\inf _{g \in \mathcal{P}_{n-1}}\left\|x^{n}-g(x)\right\|_{p, \omega}, \quad 1 \leq p<\infty .
$$

Furthermore, let $W_{n, p, \omega} \in \mathcal{P}_{n}$ satisfy

$$
\left\|W_{n, p, \omega}\right\|_{p, \omega}=E_{n, p, \omega} \quad \text { and } \quad W_{n, p, \omega}(x)=x^{n}+c_{1} x^{n-1}+\cdots+c_{n} .
$$

Then, $W_{n, p, \omega}$ is unique and has exactly $n$ zeros (see Lemma 2.2)

$$
a<\xi_{1, p, \omega}<\xi_{2, p, \omega}<\cdots<\xi_{n, p, \omega}<b
$$

Let

$$
\Delta_{n, p, \omega}=\left\{\xi_{1, p, \omega}, \xi_{2, p, \omega}, \ldots, \xi_{n, p, \omega}\right\}
$$

Then, $L_{\Delta_{n, p, \omega}}(f)$ has the explicit expression

$$
L_{\Delta_{n, p, \omega}}(f, x)=\sum_{k=1}^{n} f\left(\xi_{k, p, \omega}\right) \ell_{k, p, \omega}(x)
$$

where

$$
\ell_{k, p, \omega}(x)=\frac{W_{n, p, \omega}(x)}{\left(x-\xi_{k, p, \omega}\right) W_{n, p, \omega}^{\prime}\left(\xi_{k, p, \omega}\right)}
$$

and

$$
W_{n, p, \omega}(x)=\prod_{k=1}^{n}\left(x-\xi_{k, p, \omega}\right) .
$$


First, we obtained the following results.

\section{Theorem 1.1}

(1) For $p=\infty$, we have

$$
e\left(n, B W_{\infty}^{n}, L_{\infty}\right)=e\left(B W_{\infty}^{n}, L_{\Delta_{n, \infty}}, L_{\infty}\right)=\frac{(b-a)^{n}}{n ! 2^{2 n-1}},
$$

where

$$
\begin{aligned}
\Delta_{n, \infty}= & \left(\frac{a+b}{2}+\frac{b-a}{2} \cos \frac{(2 n-1) \pi}{2 n}, \frac{a+b}{2}+\frac{b-a}{2} \cos \frac{(2 n-3) \pi}{2 n}, \ldots,\right. \\
& \left.\frac{a+b}{2}+\frac{b-a}{2} \cos \frac{\pi}{2 n}\right) .
\end{aligned}
$$

(2) Let $1 \leq p<\infty$ and assume that $\omega(x)>0$ is continuous-integrable on $(a, b)$. Then, we have

$$
e\left(n, B W_{\infty}^{n}, L_{p, \omega}\right)=e\left(B W_{\infty}^{n}, L_{\Delta_{n, p, \omega}}, L_{p, \omega}\right)=\frac{E_{n, p, \omega}}{n !},
$$

where $\Delta_{n, p, \omega}$ is given by (1.8).

From Theorem 1.1 we know that the optimal Hermite interpolation is Lagrange interpolation, i.e., increasing the use of derivatives value data can not make the calculation result more accurate.

In practice one often wants to have boundary points as interpolation systems, i.e.,

$$
\Delta:=\left\{a=x_{1}<x_{2}<\cdots<x_{r}=b, \alpha_{i} \in \mathbb{N}, n=\sum_{i=1}^{r} \alpha_{i}\right\} .
$$

Then, the following question arises: for which $\Delta^{*}$ of the form (1.14), we have

$$
e\left(B F, H_{\Delta^{*}}, G\right)=\bar{e}(n, B F, G):=\inf _{\Delta \text { is of the form (1.14) }} e\left(B F, H_{\Delta}, G\right)
$$

For the Lagrange interpolation, Hoang [6] considered this problem for $C^{n}[-1,1]$ in $L_{\infty}[-1,1]$. Recently, Xu and Wang [16] considered this problem for $W_{\infty}^{n}[-1,1]$ in $L_{\infty}[-1,1]$ and $L_{p, \omega}[-1,1], 1 \leq p<\infty$. In this paper, we will extend the result of [16] into Hermite interpolation and obtain the following results.

\section{Theorem 1.2}

(1) Let $p=\infty$ and $n>2$. Then, we have

$$
\bar{e}\left(n, B W_{\infty}^{n}, L_{\infty}\right)=e\left(B W_{\infty}^{n}, L_{\Delta_{n, \infty}^{*}}, L_{\infty}\right)=\frac{(b-a)^{n}}{\left(\cos \frac{\pi}{2 n}\right)^{n} 2^{2 n-1} n !},
$$

where

$$
\begin{aligned}
\Delta_{n, \infty}^{*}= & \left(a, \frac{a+b}{2}+\frac{b-a}{2} \cos \frac{(2 n-3) \pi}{2 n} / \cos \frac{\pi}{2 n}, \ldots,\right. \\
& \left.\frac{a+b}{2}+\frac{b-a}{2} \cos \frac{3 \pi}{2 n} / \cos \frac{\pi}{2 n}, b\right) .
\end{aligned}
$$


(2) Let $1 \leq p<\infty, n>2$ and assume that $\omega(x)>0$ is continuous-integrable on $(a, b)$.

Then, we have

$$
\bar{e}\left(n, B W_{\infty}^{n}, L_{p, \omega}\right)=e\left(B W_{\infty}^{n}, L_{\Delta_{n, p, \omega}^{*}}, L_{p, \omega}\right)=\frac{E_{n-2, p, \bar{\omega}}}{n !},
$$

where

$$
\bar{\omega}(x)=(x-a)^{p}(b-x)^{p} \omega(x), \quad \Delta_{n, p, \omega}^{*}=\left(a, \xi_{1, p, \bar{\omega}}, \xi_{2, p, \bar{\omega}}, \ldots, \xi_{n-2, p, \bar{\omega}}, b\right)
$$

and $\xi_{1, p, \bar{\omega}}, \xi_{2, p, \bar{\omega}}, \ldots, \xi_{n-2, p, \bar{\omega}}$ are given by $(1.7)$ with $n-2$

The remainder of this paper is organized as follows. In Sect. 2, we give the proofs of Theorem 1.1 and Theorem 1.2. In Sect. 3, we give some examples to show the results.

\section{Proofs of Theorem 1.1 and Theorem 1.2}

To prove Theorem 1.1, we first give a lemma.

Lemma 2.1 Let $f \in W_{\infty}^{n}$. Assume that $\Delta:=\left\{a \leq x_{1}<x_{2}<\cdots<x_{r} \leq b, \alpha_{i} \in \mathbb{N}, n=\sum_{i=1}^{r} \alpha_{i}\right\}$ is a Hermite interpolation system. Then, the remainder $R_{\Delta}(f, x):=f(x)-H_{\Delta}(f, x)$ for the Hermite interpolation polynomial based on $\Delta$ satisfies

$$
\left|R_{\Delta}(f, x)\right|=\left|f(x)-H_{\Delta}(f, x)\right| \leq \frac{\left\|f^{(n)}\right\|_{\infty}}{n !}\left|W_{\Delta}(x)\right|, \quad x \in[a, b]
$$

where $W_{\Delta}$ is given by (1.4). In particular, iff $\in C^{n}$, then

$$
R_{\Delta}(f, x)=f(x)-H_{\Delta}(f, x)=\frac{f^{(n)}(\xi)}{n !} W_{\Delta}(x), \quad x \in[a, b]
$$

for some $\xi \in[-1,1]$ depending on $x$ and $\Delta$.

Proof Since (2.1) is trivially satisfied if $x$ coincides with one of the interpolation points $x_{1}, \ldots, x_{r}$, we need be concerned only with the case where $x$ does not coincide with one of the interpolation nodes. Keeping $x$ fixed, consider $g:[a, b] \rightarrow \mathbb{R}$ given by

$$
g(y):=R_{\Delta}(f, y)-W_{\Delta}(y) \frac{R_{\Delta}(f, x)}{W_{\Delta}(x)}, \quad y \in[a, b] .
$$

By the assumption on $f$ we know $g \in W_{\infty}^{n}$. From (1.3) and (2.3) we conclude that $g$ has at least $n+1$ zeros (counting multiplicity), namely single zero $x$ and $\alpha_{k}$ fold zeros $x_{k}, k=1, \ldots, r$. Then, by Rolle's theorem, the derivative $g^{\prime}$ has at least $n$ zeros. Repeating the argument, by induction we deduce that the derivative $g^{(n-1)}$ has at least two zeros in $[a, b]$, which we denote by $z_{1}$ and $z_{2}\left(z_{1}<z_{2}\right)$, respectively. Since $g \in W_{\infty}^{n}$, then by the Newton-Leibniz formula we obtain

$$
0=g^{(n-1)}\left(z_{2}\right)-g^{(n-1)}\left(z_{1}\right)=\int_{z_{1}}^{z_{2}} g^{(n)}(y) d y .
$$

It is known that $H_{\Delta}(f)$ is an algebraic polynomial of degree at most $n-1$. Hence, we obtain

$$
\left(H_{\Delta}(f)\right)^{(n)}(y)=0
$$


By a direct computation we obtain

$$
\left(W_{\Delta}\right)^{(n)}(y)=n ! .
$$

Substituting (2.5) and (2.6) into (2.4), we obtain

$$
0=\int_{z_{1}}^{z_{2}}\left[f^{(n)}(y)-n ! \frac{R_{\Delta}(f, x)}{W_{\Delta}(x)}\right] d y=\int_{z_{1}}^{z_{2}} f^{(n)}(y) d y-n !\left(z_{2}-z_{1}\right) \frac{R_{\Delta}(f, x)}{W_{\Delta}(x)}
$$

From (2.7) it follows that

$$
R_{\Delta}(f, x)=\frac{\int_{z_{1}}^{z_{2}} f^{(n)}(y) d y}{n !\left(z_{2}-z_{1}\right)} W_{\Delta}(x)
$$

Combining

$$
\left|\int_{z_{1}}^{z_{2}} f^{(n)}(y) d y\right| \leq \int_{z_{1}}^{z_{2}}\left|f^{(n)}(y)\right| d y \leq \int_{z_{1}}^{z_{2}}\left\|f^{(n)}\right\|_{\infty} d y=\left\|f^{(n)}\right\|_{\infty}\left(z_{2}-z_{1}\right)
$$

with (2.8) we obtain (2.1). Besides, if $f \in C^{n}$, then $g^{(n)}$ has at least one zero $\xi$ in $[a, b]$, i.e., $g^{(n)}(\xi)=0$. Hence, by differentiating $n$ times on two sides of (2.3) first, and then substituting (2.5) and (2.6) into the obtained relation, we obtain

$$
0=f^{(n)}(\xi)-n ! \frac{R_{\Delta}(f, x)}{W_{\Delta}(x)}
$$

From (2.9) we obtain (2.2). This completes the proof of Lemma 2.1.

Lemma 2.2 Let $1 \leq p<\infty$ and assume that $\omega(x)>0$ is continuous-integrable on $(-1,1)$. Then, there exists a unique $W_{n, p, \omega} \in \mathcal{P}_{n}$ for all $n \in \mathbb{N}$ such that

$$
\left\|W_{n, p, \omega}\right\|_{p, \omega}=E_{n, p, \omega} \quad \text { and } \quad W_{n, p, \omega}(x)=x^{n}+c_{1} x^{n-1}+\cdots+c_{n},
$$

where $E_{n, p, \omega}$ is given by (1.5). Furthermore, $W_{n, p, \omega}$ has exactly $n$ zeros given by (1.7).

Proof The proof of the problem on $[-1,1]$ can be found in [16]. In general, we can use the variable substitution $x=\frac{a+b}{2}+\frac{b-a}{2} t$ to refer the problem on $[a, b]$ to this on $[-1,1]$. We omit the details.

Proof of Theorem 1.1 We consider (1) first. Let $\Delta_{n, \infty}$ be given by (1.12). Then, for any $f \in W_{\infty}^{n}$, it follows from (2.1) that

$$
\begin{aligned}
& \left|f(x)-L_{\Delta_{n, \infty}}(f, x)\right| \leq \frac{\left\|f^{(n)}\right\|_{\infty}}{n !}\left|\prod_{i=1}^{n}\left(x-\frac{a+b}{2}-\frac{b-a}{2} \cos \frac{(2 i-1) \pi}{2 n}\right)\right| \\
& \quad x \in[a, b] .
\end{aligned}
$$


Let $x=\frac{a+b}{2}+\frac{b-a}{2} t$. Then, (2.10) becomes

$$
\begin{aligned}
\left|f(x)-L_{\Delta_{n, \infty}}(f, x)\right| & \leq \frac{\left\|f^{(n)}\right\|_{\infty}(b-a)^{n}}{n ! 2^{n}}\left|\prod_{i=1}^{n}\left(t-\cos \frac{(2 i-1) \pi}{2 n}\right)\right| \\
& =\frac{\left\|f^{(n)}\right\|_{\infty}(b-a)^{n}}{n ! 2^{2 n-1}}\left|T_{n}(t)\right|, \quad t \in[-1,1],
\end{aligned}
$$

where $T_{n}$ is the $n$th Chebyshev polynomial of the first kind, i.e., $T_{n}(t)=\cos (n \arccos t)$. Let $f \in B W_{\infty}^{n}$. Then, we have $\left\|f^{(n)}\right\|_{\infty} \leq 1$. Combining this fact with $\left\|T_{n}\right\|_{\infty}=1$ as well as (2.11), we obtain

$$
e\left(B W_{\infty}^{n}, L_{\Delta_{n, \infty}}, L_{\infty}\right)=\sup _{f \in B W_{\infty}^{n}}\left\|f-L_{\Delta_{n, \infty}}(f)\right\|_{\infty} \leq \frac{(b-a)^{n}}{n ! 2^{2 n-1}} .
$$

From (1.2) and (2.12) we obtain the upper estimate.

Now, we consider the lower estimate. Let $\Delta:=\left\{a \leq x_{1}<x_{2}<\cdots<x_{r} \leq b, \alpha_{i} \in \mathbb{N}, n=\right.$ $\sum_{i=1}^{r} \alpha_{i}$ \} be an arbitrary Hermite interpolation system of cardinality $n$ in $[a, b]$. Consider the function $g(x)=\frac{x^{n}}{n !}$. Then, from $g^{(n)}(x)=1$ and (2.2) it follows that $g \in W_{\infty}^{n}$ and

$$
g(x)-H_{\Delta}(g, x)=\frac{W_{\Delta}(x)}{n !}, \quad x \in[a, b]
$$

Let $x=\frac{a+b}{2}+\frac{b-a}{2} t$. Then, by (1.4) we obtain

$$
W_{\Delta}(x)=x^{n}+a_{1} x^{n-1}+a_{2} x^{n-2}+\cdots+a_{n}=\frac{(b-a)^{n}}{2^{n}} h(t), \quad t \in[-1,1],
$$

where

$$
h(t)=t^{n}+b_{1} t^{n-1}+b_{2} t^{n-2}+\cdots+b_{n} .
$$

Then, it follows from Theorem 6.1 in [4, Ch. 3] that

$$
\|h\|_{\infty} \geq 2^{1-n}
$$

Combining (1.1), (2.13), (2.14) and (2.16), we obtain

$$
e\left(B W_{\infty}^{n}, H_{\Delta}, L_{\infty}\right) \geq\left\|g-H_{\Delta}(g)\right\|_{\infty}=\frac{\left\|W_{\Delta}\right\|_{\infty}}{n !}=\frac{(b-a)^{n}}{n ! 2^{n}}\|h\|_{\infty} \geq \frac{(b-a)^{n}}{n ! 2^{2 n-1}} .
$$

From (1.2) and (2.17) we obtain the lower estimate.

Next, we consider (2). We consider the upper estimate first. Let $\Delta_{n, p, \omega}$ be given by (1.8) and $W_{n, p, \omega}$ be given by (1.6). If $f \in B W_{\infty}^{n}$, then we have $\left\|f^{(n)}\right\|_{\infty} \leq 1$. Combining this fact with (2.1) we obtain

$$
\left|f(x)-L_{\Delta_{n, p, \omega}}(f, x)\right| \leq \frac{\left|W_{n, p, \omega}(x)\right|}{n !}, \quad x \in[a, b]
$$

It follows that

$$
\left\|f-L_{\Delta_{n, p, \omega}}(f)\right\|_{p, \omega} \leq \frac{\left\|W_{n, p, \omega}\right\|_{p, \omega}}{n !}=\frac{E_{n, p, \omega}}{n !} .
$$


From (1.1) and (2.18) we obtain

$$
e\left(B W_{\infty}^{n}, L_{\Delta_{n, p, \omega}}, L_{p, \omega}\right) \leq \frac{E_{n, p, \omega}}{n !}
$$

From (1.2) and (2.19) we obtain the upper estimate.

Now, we consider the lower estimate. Let $\Delta:=\left\{a \leq x_{1}<x_{2}<\cdots<x_{r} \leq b, \alpha_{i} \in \mathbb{N}, n=\right.$ $\sum_{i=1}^{r} \alpha_{i}$ \} be an arbitrary Hermite interpolation system of cardinality $n$ in $[a, b]$. Consider the function $g(x)=\frac{x^{n}}{n !}$. Then, $g \in W_{\infty}^{n}$ and (2.13) holds. From the first equality in (2.14) and (1.5) as well as (1.6) it follows that

$$
\left\|W_{\Delta}\right\|_{p, \omega} \geq E_{n, p, \omega}
$$

From (1.1), (2.13) and (2.20) it follows that

$$
e\left(B W_{\infty}^{n}, H_{\Delta}, L_{p, \omega}\right) \geq\left\|g-H_{\Delta}(g)\right\|_{p, \omega}=\frac{\left\|W_{\Delta}\right\|_{p, \omega}}{n !} \geq \frac{E_{n, p, \omega}}{n !} .
$$

From (1.2) and (2.21) we obtain the lower estimate of (2). Theorem 1.1 is proved.

Let $B C^{n}=\left\{f \in C^{n}:\left\|f^{(n)}\right\|_{\infty} \leq 1\right\}$. Using the fact $B W_{\infty}^{n} \subset B C^{n}$ and $g(x)=\frac{x^{n}}{n !} \in B C^{n}$ for $n \in \mathbb{N}$, combining the proof of Theorem 1.1, we obtained the following results.

\section{Corollary 2.3}

(1) For $p=\infty$, we have

$$
e\left(n, B C^{n}, L_{\infty}\right)=e\left(B C^{n}, L_{\Delta_{n, \infty}}, L_{\infty}\right)=\frac{(b-a)^{n}}{n ! 2^{2 n-1}},
$$

where $\Delta_{n, \infty}$ is given by (1.12).

(2) Let $1 \leq p<\infty$ and assume that $\omega(x)>0$ is continuous-integrable on $(a, b)$. Then, we have

$$
e\left(n, B C^{n}, L_{p, \omega}\right)=e\left(B C^{n}, L_{\Delta_{n, p, \omega}}, L_{p, \omega}\right)=\frac{E_{n, p, \omega}}{n !},
$$

where $\Delta_{n, p, \omega}$ is given by (1.8).

Proof of Theorem 1.2 We consider (1) first. For any $f \in B W_{\infty}^{n}$, from (2.1) it follows that

$$
\begin{aligned}
& \left|f(x)-L_{\Delta_{n, \infty}^{*}}(f, x)\right| \leq \frac{1}{n !}\left|\prod_{i=1}^{n}\left(x-\frac{a+b}{2}-\frac{b-a}{2} \cos \frac{(2 i-1) \pi}{2 n} / \cos \frac{\pi}{2 n}\right)\right|, \\
& \quad x \in[a, b] .
\end{aligned}
$$

Let $x=\frac{a+b}{2}+\frac{b-a}{2 \cos \frac{\pi}{2 n}} t$. Then, we have

$$
\begin{aligned}
& \prod_{i=1}^{n}\left(x-\frac{a+b}{2}-\frac{b-a}{2} \cos \frac{(2 i-1) \pi}{2 n} / \cos \frac{\pi}{2 n}\right)=\frac{(b-a)^{n} T_{n}(t)}{\left(\cos \frac{\pi}{2 n}\right)^{n} 2^{2 n-1}} \\
& t \in\left[-\cos \frac{\pi}{2 n}, \cos \frac{\pi}{2 n}\right] .
\end{aligned}
$$


From (1.1), (2.24) and (2.25) it follows that

$$
\begin{aligned}
e\left(B W_{\infty}^{n}, L_{\Delta_{n, \infty}^{*}}, L_{\infty}\right) & \leq \frac{(b-a)^{n}}{\left(\cos \frac{\pi}{2 n}\right)^{n} 2^{2 n-1} n !} \sup _{t \in\left[-\cos \frac{\pi}{2 n}, \cos \frac{\pi}{2 n}\right]}\left|T_{n}(t)\right| \\
& =\frac{(b-a)^{n}}{\left(\cos \frac{\pi}{2 n}\right)^{n} 2^{2 n-1} n !}
\end{aligned}
$$

From (1.2) and (2.26) we obtain the upper estimate.

Now, we consider the lower estimate. Let $\Delta:=\left\{a=x_{1}<x_{2}<\cdots<x_{r}=b, \alpha_{i} \in \mathbb{N}, n=\right.$ $\left.\sum_{i=1}^{r} \alpha_{i}\right\}$ be an arbitrary Hermite interpolation system of cardinality $n$ including the endpoints $a$ and $b$. Consider the function $g(x)=\frac{x^{n}}{n !}$. Then, $g \in W_{\infty}^{n}$ and (2.13) holds. Let $x=\frac{a+b}{2}+\frac{b-a}{2} t$. Denote $t_{i}=\frac{2}{b-a}\left(x_{i}-\frac{a+b}{2}\right), i=1, \ldots, r$. Then, by (1.4) one obtains

$$
W_{\Delta}(x)=\frac{(b-a)^{n}}{2^{n}} \prod_{i=1}^{r}\left(t-t_{i}\right)^{\alpha_{i}}, \quad t_{1}=-1, t_{r}=1, t \in[-1,1] .
$$

Let

$$
g(t)=\left(t^{2}-1\right) \prod_{i=2}^{n-1}\left(t-\cos \frac{(2 i-1) \pi}{2 n} / \cos \frac{\pi}{2 n}\right)=\frac{T_{n}\left(t \cos \frac{\pi}{2 n}\right)}{2^{n-1}\left(\cos \frac{\pi}{2 n}\right)^{n}}
$$

Then, it is easy to verify that

$$
\|g\|_{\infty}=\frac{1}{2^{n-1}\left(\cos \frac{\pi}{2 n}\right)^{n}}
$$

and

$$
g\left(\frac{\cos \frac{i \pi}{n}}{\cos \frac{\pi}{2 n}}\right)=\frac{(-1)^{i}}{2^{n-1}\left(\cos \frac{\pi}{2 n}\right)^{n}}, \quad i=1, \ldots, n-1
$$

Assume that

$$
\left\|\prod_{i=1}^{r}\left(t-t_{i}\right)^{\alpha_{i}}\right\|_{\infty}<\frac{1}{2^{n-1}\left(\cos \frac{\pi}{2 n}\right)^{n}} .
$$

Let

$$
R(t)=g(t)-\prod_{i=1}^{r}\left(t-t_{i}\right)^{\alpha_{i}}, \quad t \in[-1,1]
$$

Then, it is easy to verify that $R(t)$ is a polynomial of degree at most $n-1$. Furthermore, from (2.29) and (2.30) one can check that

$$
R\left(\frac{\cos \frac{i \pi}{n}}{\cos \frac{\pi}{2 n}}\right)(-1)^{i}>0, \quad i=1, \ldots, n-1
$$

Thus, the polynomial $R(t)$ has at least $n-2$ zeros in $(-1,1)$. As $t_{1}=-1, t_{r}=1$, it is clear that \pm 1 are zeros of $R(t)$. Hence, $R(t)$ has at least $n$ zeros in $[-1,1]$. This, and the fact that 
$R(t)$ is a polynomial of degree at most $n-1$, implies that $R(t)=0$. Therefore,

$$
\left\|\prod_{i=1}^{r}\left(t-t_{i}\right)^{\alpha_{i}}\right\|_{\infty}=\|g\|_{\infty}=\frac{1}{2^{n-1}\left(\cos \frac{\pi}{2 n}\right)^{n}},
$$

which contradicts (2.30). Hence, we have

$$
\left\|\prod_{i=1}^{r}\left(t-t_{i}\right)^{\alpha_{i}}\right\|_{\infty} \geq \frac{1}{2^{n-1}\left(\cos \frac{\pi}{2 n}\right)^{n}} .
$$

From (1.1), (2.13), (2.27) and (2.31) we obtain

$$
e\left(B W_{\infty}^{n}, H_{\Delta}, L_{\infty}\right) \geq\left\|g-H_{\Delta}(g)\right\|_{\infty}=\frac{\left\|W_{\Delta}\right\|_{\infty}}{n !} \geq \frac{(b-a)^{n}}{\left(\cos \frac{\pi}{2 n}\right)^{n} 2^{2 n-1} n !} .
$$

From (1.2) and (2.32) we obtain the lower estimate of (1).

Next, we consider (2). Let $\bar{\omega}$ and $\Delta_{n, p, \omega}^{*}$ be given by (1.19). Then, for any $f \in B W_{\infty}^{n}$, from (2.1) it follows that

$$
\left|f(x)-L_{\Delta_{n, p, \omega}^{*}}(f, x)\right| \leq \frac{\left(1-x^{2}\right)\left|W_{n-2, p, \bar{\omega}}(x)\right|}{n !}, \quad x \in[a, b] .
$$

From (2.33) it follows that

$$
\left\|f-L_{\Delta_{n, p, \omega}^{*}}(f)\right\|_{p, \omega} \leq \frac{\left\|W_{n-2, p, \bar{\omega}}\right\|_{p, \bar{\omega}}}{n !}=\frac{E_{n-2, p, \bar{\omega}}}{n !} .
$$

From (1.1) and (2.34) we conclude that

$$
e\left(B W_{\infty}^{n}, L_{\Delta_{n, p, \omega}^{*}}, L_{p, \omega}\right) \leq \frac{E_{n-2, p, \bar{\omega}}}{n !} .
$$

On the other hand, let $\Delta:=\left\{a=x_{1}<x_{2}<\cdots<x_{r}=b, \alpha_{i} \in \mathbb{N}, n=\sum_{i=1}^{r} \alpha_{i}\right\}$ be an arbitrary Hermite interpolation system of cardinality $n$ including the endpoints. Consider the function $g(x)=\frac{x^{n}}{n !}$. Then, $g \in W_{\infty}^{n}$ and (2.13) holds. From (1.1), (2.13), (1.5) and (1.6) it follows that

$$
\begin{aligned}
e\left(B W_{\infty}^{n}, H_{\Delta}, L_{p, \omega}\right) & \geq\left\|g-H_{\Delta}(g)\right\|_{p, \omega}=\frac{1}{n !}\left\|\prod_{k=1}^{r}\left(x-x_{k}\right)^{\alpha_{k}}\right\|_{p, \omega} \\
& =\frac{1}{n !}\left\|(x-a)^{\alpha_{1}-1}(b-x)^{\alpha_{r}-1} \prod_{k=2}^{r-1}\left(x-x_{k}\right)^{\alpha_{k}}\right\|_{p, \bar{\omega}} \\
& \geq \frac{1}{n !}\left\|\prod_{k=1}^{n-2}\left(x-\xi_{k, p, \bar{\omega}}\right)\right\|_{p, \bar{\omega}}=\frac{E_{n-2, p, \bar{\omega}}}{n !} .
\end{aligned}
$$

From (2.35) and (2.36) as well as (1.2) we obtain the result of (2). Theorem 1.2 is proved.

Using the fact that $B C^{n} \subset B W_{\infty}^{n}$ and $g(x)=\frac{x^{n}}{n !} \in B C^{n}$ for $n \in \mathbb{N}$, combining the proof of Theorem 1.2, we obtained the following results. 


\section{Corollary 2.4}

(1) Let $p=\infty$ and $n>2$. Then, we have

$$
\bar{e}\left(n, B C^{n}, L_{\infty}\right)=e\left(B C^{n}, L_{\Delta_{n, \infty}^{*}}, L_{\infty}\right)=\frac{(b-a)^{n}}{\left(\cos \frac{\pi}{2 n}\right)^{n} 2^{2 n-1} n !},
$$

where $\Delta_{n, \infty}^{*}$ is given by (1.17).

(2) Let $1 \leq p<\infty, n>2$ and assume that $\omega(x)>0$ is continuous-integrable on $(a, b)$.

Then, we have

$$
\bar{e}\left(n, B C^{n}, L_{p, \omega}\right)=e\left(B C^{n}, L_{\Delta_{n, p, \omega}^{*}}, L_{p, \omega}\right)=\frac{E_{n-2, p, \bar{\omega}}}{n !}
$$

where $\bar{\omega}$ and $\Delta_{n, p, \omega}^{*}$ are given by (1.19).

Remark 2.5 When $n \neq r$, the $n$th optimal Hermite interpolation system of the problems given by (1.2) and (1.15) for $B W_{\infty}^{r}$ in $L_{\infty}$ and $L_{p, \omega}(1 \leq p<\infty)$ are open problems.

Remark 2.6 When $n=r$, the $n$th optimal Birkhoff interpolation system of the problems given by (1.2) and (1.15) for $B W_{\infty}^{n}$ in $L_{\infty}$ and $L_{p, \omega}(1 \leq p<\infty)$ are open problems.

\section{Some examples}

Example 1 Let $\omega(x)=1,[a, b]=[-1,1]$. Then for $1 \leq p<\infty$ we obtain the usual $L_{p} \equiv$ $L_{p}[-1,1]$ spaces. For $p=1$, it follows from $[4$, pp. 87-88] that

$$
E_{n, 1,1}=\frac{1}{2^{n-1}}, \quad W_{n, 1,1}(x)=\frac{U_{n}(x)}{2^{n}}, \quad \xi_{k, 1,1}=\cos \frac{k \pi}{n+1}, \quad k=1, \ldots, n,
$$

where $U_{n}$ is the $n$th Chebyshev polynomial of the second kind, i.e.,

$$
U_{n}(x)=\frac{\sin (n+1) \theta}{\sin \theta}, \quad x=\cos \theta .
$$

From Theorem 1.1 it follows that

$$
e\left(n, B W_{\infty}^{n}, L_{1}\right)=e\left(B W_{\infty}^{n}, L_{\Delta_{n, 1,1}}, L_{1}\right)=\frac{E_{n, 1,1}}{n !}=\frac{1}{2^{n-1} n !},
$$

where $\Delta_{n, 1,1}=\left\{\cos \frac{n \pi}{n+1}, \cos \frac{(n-1) \pi}{n+1}, \ldots, \cos \frac{\pi}{n+1}\right\}$.

Example 2 Let $p=2$. In this case, for any continuous-integrable weight function $\omega(x)>0$ on $(a, b)$, there is a unique orthogonal system $\left\{p_{k, \omega}\right\}_{k \in \mathbb{Z}_{+}}$in $L_{2, \omega}$ that is complete and satisfies the following conditions:

(1) $p_{k, \omega} \in \mathcal{P}_{k}$ for all $k \in \mathbb{Z}_{+}$.

(2)

$$
\int_{a}^{b} p_{k, \omega}(x) p_{j, \omega}(x) \omega(x) d x= \begin{cases}0, & k \neq j \\ 1, & k=j\end{cases}
$$

(3) The coefficient $C_{k, \omega}$ of the leading term $x^{k}$ of $p_{k, \omega}$ is positive. 
In this case, similar to (3.18) in [16], we have $W_{k, 2, \omega}=\frac{p_{k, \omega}}{C_{k, \omega}}$ and

$$
E_{k, 2, \omega}=\left\|W_{k, 2, \omega}\right\|_{2, \omega}=1 / C_{k, \omega} .
$$

Let $\omega^{(\alpha, \beta)}$ be the Jacobi weights, i.e., $\omega^{(\alpha, \beta)}(x)=(1-x)^{\alpha}(1+x)^{\beta}$ with $\alpha, \beta>-1$ on $(-1,1)$ and we denote the corresponding orthogonal system as $\left\{p_{k}^{(\alpha, \beta)}\right\}_{k \in \mathbb{Z}_{+}}$. It is known that the coefficient of the leading term $x^{k}$ of $p_{k}^{(\alpha, \beta)}(x)$ is (see (3.21) in [16])

$$
C_{k, \omega}^{(\alpha, \beta)}=\frac{\sqrt{\alpha+\beta+2 k+1} \Gamma(\alpha+\beta+2 k+1)}{2^{k+(\alpha+\beta+1) / 2} \sqrt{k ! \Gamma(\alpha+\beta+k+1) \Gamma(\alpha+k+1) \Gamma(\beta+k+1)}} .
$$

From Theorem 1.1, (3.2) and (3.3), it follows that

$$
\begin{aligned}
& e\left(n, B W_{\infty}^{n}, L_{2, \omega}^{(\alpha, \beta)}\right)=e\left(B W_{\infty}^{n}, L_{\Delta_{n, 2, \omega}(\alpha, \beta)}, L_{2, \omega}(\alpha, \beta)\right)=\frac{1}{n ! C_{n, \omega^{(\alpha, \beta)}}} \\
& =\frac{2^{n+(\alpha+\beta+1) / 2} \sqrt{\Gamma(\alpha+\beta+n+1) \Gamma(\alpha+n+1) \Gamma(\beta+n+1)}}{\sqrt{n !(\alpha+\beta+2 n+1)} \Gamma(\alpha+\beta+2 n+1)},
\end{aligned}
$$

where $\Delta_{n, 2, \omega^{(\alpha, \beta)}}$ consists of the zeros of $p_{n}^{(\alpha, \beta)}$. From Theorem 1.2, (3.2), (3.3) and (3.4), it follows that for $n>2$

$$
\begin{aligned}
& \bar{e}\left(n, B W_{\infty}^{n}, L_{2, \omega}(\alpha, \beta)\right)=e\left(B W_{\infty}^{n}, L_{\Delta_{n, 2, \omega}^{*}(\alpha, \beta)}, L_{2, \omega^{(\alpha, \beta)}}\right) \\
& =\frac{E_{n-2,2, \omega^{(\alpha+2, \beta+2)}}}{n !}=\frac{1}{n ! C_{n-2, \omega^{(\alpha+2, \beta+2)}}} \\
& =\sqrt{\frac{(\alpha+\beta+n+2)(\alpha+\beta+n+1)}{n(n-1)}} e\left(n, B W_{\infty}^{n}, L_{2, \omega}(\alpha, \beta)\right) \text {, }
\end{aligned}
$$

where $\Delta_{n, 2, \omega^{(\alpha, \beta)}}^{*}=\left(-1, \xi_{1,2, \omega^{(\alpha+2, \beta+2)}}, \xi_{2,2, \omega^{(\alpha+2, \beta+2)}}, \ldots, \xi_{n-2,2, \omega^{(\alpha+2, \beta+2)}}, 1\right)$, and $\xi_{1,2, \omega^{(\alpha+2, \beta+2)}}$,

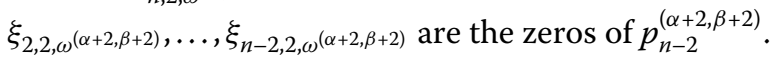

Next, we list three examples.

For $\alpha=\beta=0$, i.e., $\omega^{(0,0)}(x)=1$, we have (see [7, p. 205])

$$
W_{n, 2,1}(x)=\frac{2^{n}(n !)^{2}}{(2 n) !} P_{n}(x)=\prod_{k=1}^{n}\left(x-\xi_{k, 2,1}\right)
$$

where $P_{n}$ is the $n$th Legendre polynomial, i.e.,

$$
P_{n}(x)=\frac{1}{2^{n} n !} \frac{d^{n}}{d x^{n}}\left(x^{2}-1\right)^{n}
$$

From (3.4) it follows that

$$
e\left(n, B W_{\infty}^{n}, L_{2}\right)=e\left(B W_{\infty}^{n}, L_{\Delta_{n, 2,1}}, L_{2}\right)=\frac{2^{n+1 / 2} n !}{(2 n) ! \sqrt{2 n+1}}
$$


where $\Delta_{n, 2,1}$ consists of the zeros of $P_{n}$. Furthermore, from (3.5) and (3.7) it follows that for $n>2$

$$
\bar{e}\left(n, B W_{\infty}^{n}, L_{2}\right)=\frac{2^{n+1 / 2}(n+2) !}{(2 n) ! \sqrt{(n-1) n(n+1)(n+2)(2 n+1)}} .
$$

For $\alpha=\beta=-1 / 2$, i.e., $\omega^{(-1 / 2,-1 / 2)}(x)=\frac{1}{\sqrt{1-x^{2}}}$, we know $W_{n, 2, \omega(-1 / 2,-1 / 2)}(x)=\frac{T_{n}(x)}{2^{n-1}}$. From (3.4) it follows that

$$
e\left(n, B W_{\infty}^{n}, L_{2, \omega}(-1 / 2,-1 / 2)\right)=e\left(B W_{\infty}^{n}, L_{\Delta_{n, 2, \omega}(-1 / 2,-1 / 2)}, L_{2, \omega}(-1 / 2,-1 / 2)\right)=\frac{\sqrt{2 \pi}}{2^{n} n !},
$$

where $\Delta_{n, 2, \omega(-1 / 2,-1 / 2)}=\left\{\cos \frac{(2 n-1) \pi}{2 n}, \cos \frac{(2 n-3) \pi}{2 n}, \ldots, \cos \frac{\pi}{2 n}\right\}$. Furthermore, from (3.5) and (3.9) it follows that for $n>2$

$$
\bar{e}\left(n, B W_{\infty}^{n}, L_{2, \omega^{(-1 / 2,-1 / 2)}}\right)=\frac{\sqrt{2 \pi(n+1)(n-1)}}{2^{n}(n-1) n !} .
$$

For $\alpha=\beta=1 / 2$, i.e., $\omega^{(1 / 2,1 / 2)}(x)=\sqrt{1-x^{2}}$, we know $W_{n, 2, \omega}^{(1 / 2,1 / 2)}(x)=\frac{U_{n}(x)}{2^{n}}$. From (3.4) it follows that

$$
e\left(n, B W_{\infty}^{n}, L_{2, \omega}^{(1 / 2,1 / 2)}\right)=e\left(B W_{\infty}^{n}, L_{\Delta_{n, 2, \omega}(1 / 2,1 / 2)}, L_{2, \omega(1 / 2,1 / 2)}\right)=\frac{\sqrt{\pi}}{2^{n+1 / 2} n !},
$$

where $\Delta_{n, 2, \omega}(1 / 2,1 / 2)=\Delta_{n, 1,1}$. From (3.5) and (3.10) it follows that for $n>2$

$$
\bar{e}\left(n, B W_{\infty}^{n}, L_{2, \omega}^{(1 / 2,1 / 2)}\right)=\frac{\sqrt{\pi(n+3)(n+2) n(n-1)}}{2^{n+1 / 2} n ! n(n-1)} .
$$

\section{Acknowledgements}

Not applicable.

Funding

This work was supported by the National Natural Science Foundation of China $(11871006,11671271)$.

Availability of data and materials

Not applicable.

\section{Declarations}

Competing interests

The authors declare that they have no competing interests.

\section{Authors' contributions}

XG constructed the outline of the paper, YX completed the details of the paper. All authors read and approved the final manuscript.

\section{Authors' information}

Xu Guiqiao, male, professor, the winner of THE 2020 JOSEPH F. TRAUB PRIZE FOR ACHIEVEMENT IN INFORMATION-BASED COMPLEXITY. Yu Xiaochen, female, postgraduate.

\section{Publisher's Note}

Springer Nature remains neutral with regard to jurisdictional claims in published maps and institutional affiliations.

Received: 8 October 2020 Accepted: 22 December 2021 Published online: 04 January 2022 
References

1. Babaev, S.S., Hayotov, A.R.: Optimal interpolation formulas in $W_{2}^{(m, m-1)}$ space. Calcolo 56, $23-45$ (2019)

2. Barthelmann, V., Novak, E., Ritter, K.: High dimensional polynomial interpolation on sparse grids. Adv. Comput. Math. $12,273-288(2000)$

3. Ben, A., Yi, S.: Compressive Hermite interpolation: sparse, high-dimensional approximation from gradient-augmented measurements. Constr. Approx. 50, 167-207 (2019)

4. Devore, R.A., Lorentz, G.G.: Constructive Approximation. Springer, New York (1993)

5. Hinrichs, A., Novak, E., Ullrich, M.: On weak tractability of the Clenshaw-Curtis Smolyak algorithm. J. Approx. Theory 183, 31-44 (2014)

6. Hoang, N.S.: On node distributions for interpolation and spectral methods. Math. Comput. 85, 667-692 (2016)

7. Kress, R.: Numerical Analysis. Springer, New York (2003)

8. Liu, Y.P., Xu, G.Q., Zhang, J.: Exponential convergence of an approximation problem for infinitely differentiable multivariate functions. Math. Notes 103, 769-779 (2018)

9. Mastroianni, G., Occorsio, D.: Optimal systems of nodes for Lagrange interpolation on bounded intervals. A survey. J. Comput. Appl. Math. 134, 325-341 (2001)

10. Peng, J., Hampton, J., Doostan, A.: On polynomial chaos expansion via gradient-enhanced $\ell_{1}$ minimization. J. Comput. Phys. 310, 440-458 (2016)

11. Rack, H.J., Vajda, R.: On optimal quadratic Lagrange interpolation: extremal node systems with minimal Lebesgue constant via symbolic computation. Serdica J. Comput. 8, 71-96 (2014)

12. Rack, H.J., Vajda, R.: Optimal cubic Lagrange interpolation: extremal node systems with minimal Lebesgue constant. Stud. Univ. Babeş-Bolyai, Math. 60(2), 151-171 (2015)

13. Vybíral, J.: Weak and quasi-polynomial tractability of approximation of infinitely differentiable functions. J. Complex. 30, 48-55 (2014)

14. Wasilkowski, G.W.: Tractability of approximation of $\infty$-variate functions with bounded mixed partial derivatives. J. Complex. 30, 325-346 (2014)

15. Xu, G.Q.: On weak tractability of the Smolyak algorithm for approximation problems. J. Approx. Theory 192, 347-361 (2015)

16. Xu, G.Q., Wang, H.: Sample numbers and optimal Lagrange interpolation in Sobolev spaces. Rocky Mt. J. Math. 51(1), 347-361 (2021)

\section{Submit your manuscript to a SpringerOpen ${ }^{\circ}$ journal and benefit from:}

- Convenient online submission

- Rigorous peer review

Open access: articles freely available online

- High visibility within the field

- Retaining the copyright to your article

Submit your next manuscript at $\boldsymbol{~ s p r i n g e r o p e n . c o m ~}$ 